\title{
The applications of DNA methylation as a biomarker in kidney transplantation: a systematic review
}

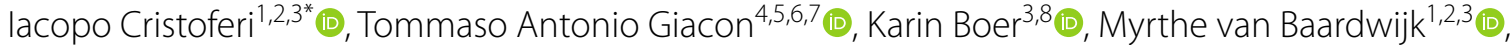

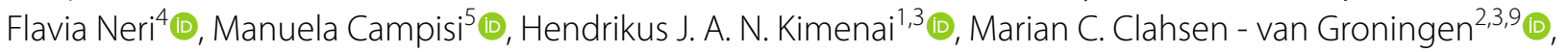 \\ Sofia Pavanello ${ }^{5}$, Lucrezia Furian ${ }^{4}$ (D) and Robert C. Minnee ${ }^{1,3}$ (I)
}

\begin{abstract}
Background: Although kidney transplantation improves patient survival and quality of life, long-term results are hampered by both immune- and non-immune-mediated complications. Current biomarkers of post-transplant complications, such as allograft rejection, chronic renal allograft dysfunction, and cutaneous squamous cell carcinoma, have a suboptimal predictive value. DNA methylation is an epigenetic modification that directly affects gene expression and plays an important role in processes such as ischemia/reperfusion injury, fibrosis, and alloreactive immune response. Novel techniques can quickly assess the DNA methylation status of multiple loci in different cell types, allowing a deep and interesting study of cells' activity and function. Therefore, DNA methylation has the potential to become an important biomarker for prediction and monitoring in kidney transplantation.

Purpose of the study: The aim of this study was to evaluate the role of DNA methylation as a potential biomarker of graft survival and complications development in kidney transplantation.

Material and Methods: A systematic review of several databases has been conducted. The Newcastle-Ottawa scale and the Jadad scale have been used to assess the risk of bias for observational and randomized studies, respectively.

Results: Twenty articles reporting on DNA methylation as a biomarker for kidney transplantation were included, all using DNA methylation for prediction and monitoring. DNA methylation pattern alterations in cells isolated from different tissues, such as kidney biopsies, urine, and blood, have been associated with ischemia-reperfusion injury and chronic renal allograft dysfunction. These alterations occurred in different and specific loci. DNA methylation status has also proved to be important for immune response modulation, having a crucial role in regulatory T cell definition and activity. Research also focused on a better understanding of the role of this epigenetic modification assessment for regulatory $T$ cells isolation and expansion for future tolerance induction-oriented therapies.
\end{abstract}

Conclusions: Studies included in this review are heterogeneous in study design, biological samples, and outcome. More coordinated investigations are needed to affirm DNA methylation as a clinically relevant biomarker important for prevention, monitoring, and intervention.

Keywords: Systematic review, Kidney transplantation, DNA methylation, Biomarker, Reperfusion injury

${ }^{*}$ Correspondence: i.cristoferi@erasmusmc.nl

${ }^{1}$ Division of HPB and Transplant Surgery, Department of Surgery, Erasmus MC, University Medical Center Rotterdam, Doctor Molewaterplein 40, 3015GD Rotterdam, the Netherlands

Full list of author information is available at the end of the article

\section{Background}

Kidney transplantation is the treatment of choice for patients undergoing end-stage renal failure [1] and improves survival and quality of life. A clear amelioration original author(s) and the source, provide a link to the Creative Commons licence, and indicate if changes were made. The images or other third party material in this article are included in the article's Creative Commons licence, unless indicated otherwise in a credit line to the material. If material is not included in the article's Creative Commons licence and your intended use is not permitted by statutory regulation or exceeds the permitted use, you will need to obtain permission directly from the copyright holder. To view a copy of this licence, visit http://creativecommons.org/licenses/by/4.0/. The Creative Commons Public Domain Dedication waiver (http://creativeco mmons.org/publicdomain/zero/1.0/) applies to the data made available in this article, unless otherwise stated in a credit line to the data. 
in short-term outcomes has been observed in the last decades, while a proportional improvement in longterm results is still missing because of the immune- and non-immune-mediated complications that affect these outcomes [2-8]. Efforts have been made to improve preventive measures and optimize treatment. Along with this, the identification of patients with a higher risk of post-transplant complications is of great importance. Current biomarkers of post-transplant complications and survival include epitope mismatch [9] and anti-HLA antibodies [10]. Their predictive value is suboptimal, raising the need to explore novel approaches for the management of transplant patients [11-13].

Epigenetic modifications get a lot of interest as a novel biomarker in transplantation. These modifications are reversible changes to the genome that occur without any alteration in the DNA sequence. The three main epigenetic modifications are histone modification, DNA methylation, and nucleosome positioning [14]. DNA methylation consists in the formation of a covalent bond between a methyl group and a cytosine almost exclusively in the context of cytosine-phosphate-guanine (CpG) dinucleotides, often clustered in regions called CpG islands [15] that are associated with about $60 \%$ of human genes promoters [16]. DNA methylation is generally associated with gene silencing, primarily affecting transcription [15]. Epigenetic mechanisms play an important role in multiple biological events involved in post-transplant complications development, such as the alloreactive immune response [17-21], ischemia/reperfusion injury (IRI) [22-24], and kidney graft fibrosis [7, 25-30]. DNA methylation assessment of specific loci is also crucial for the evaluation of biological or epigenetic age (DNAmAge) using epigenetic clocks [31-33].

Considering the central role that big data analysis is having in every research field and the new methylationwide assessment technologies that have been developed, DNA methylation has the potential to become an important biomarker for prediction and monitoring in kidney transplantation, and its use could become pivotal for the development of new therapeutic strategies [7, 34, 35]. Therefore, we performed a systematic review to evaluate the status of research concerning the role of DNA methylation as a biomarker in kidney transplantation.

\section{Materials and methods}

This systematic review was performed according to the guidelines for observational studies as described in the Preferred Reporting Items for Systematic Reviews and Meta-Analyses (PRISMA) statement [36, 37]. The data extraction and results exposition of this review have been organized into two major topics: I ischemia-reperfusion injury, fibrosis, and long-term complications-related studies and II immune response modulation-related studies.

\section{Search strategy}

With the help of a clinical librarian, we searched EMBASE, Medline ALL Ovid, Web of Science, Cochrane CENTRAL Register of Trials, and Google Scholar databases. The search terms for the other databases have been created starting from the EMBASE database search. The search included the following terms: DNA methylation, hypermethylation, hypomethylation, demethylation combined with kidney, renal transplantation, graft, allograft, allotransplantation, fibrosis, recipient, failure, reperfusion, and insufficiency. The databases have been searched from inception to September 30, 2021. For all the articles reaching the full-text-reading phase of the selection, references have been manually checked. Detailed search strategies are included in Additional file 1: Table S1.

\section{Study selection}

The studies were initially reviewed, screening title and abstract, by two independent reviewers (IC and TAG). The following inclusion criteria were applied: original articles (not reviews, editorials, or conference abstracts); English language; working on human samples; study focused on DNA methylation in kidney donors or kidney transplant recipients; at least one DNA methylation assessment performed. No restrictions have been used for study designs, population characteristics, and the number of included subjects. Important exclusion criteria have been used: not focusing on transplantation; focusing on general transplantation or combined transplantation with no possibility to extrapolate kidney-specific data; working only with samples of animal origin; not assessing DNA methylation. Disagreements were discussed between both reviewers and, when necessary, with a third party (RCM).

\section{Risk of bias assessment}

For non-randomized trials, the Newcastle-Ottawa scale $[38,39]$ has been used to assess the risk of bias. For clinical trials, the expanded six-point version of the Jadad scale [40] has been used to assess appropriate randomization, blinding, and management of withdrawals and dropouts.

Risk of bias has been assessed by two independent reviewers (IC and TAG), and disagreements were discussed between them and, when necessary, with a third party (RCM).

\section{Data collection and extraction}

A data extraction sheet has been developed, and the following features have been extracted from each study: 
research group, year of publication, country, study design, study's aim, study population, results, sample tissue, extent of the methylation assessment, bisulfite conversion, methylation assessment method, methylation outcome, statistical tests, and statistical thresholds.

\section{Results}

A total number of 4455 potentially relevant studies were identified. Figure 1 presents the PRISMA flow diagram. Twenty studies met the inclusion criteria and were included in the qualitative synthesis. The characteristics of the included studies are summarized in two tables (Tables 1,2 ) divided into the two major topics: I ischemia-reperfusion injury, fibrosis, and longterm complications-related studies; II immune response modulation-related studies. The methodology, the statistical analysis, and the identified candidate genes of the included studies are summarized in Table 3. Figure 2 represents an overview of the summarization strategy and the main findings of this systematic review. The included studies were conducted between 2006 and 2021 in ten different countries. Study sample size ranged from 9 to 188 , with a mean size of 72.3 (in two studies, only the number of biopsies was provided). Fourteen studies worked on blood samples, five studies used kidney biopsies, and urine has been used by a single study. For what concerns DNA methylation analysis design, six studies performed only genome-wide analysis, 11 studies performed only candidate genes analysis, among which eight studies investigated the methylation status of the

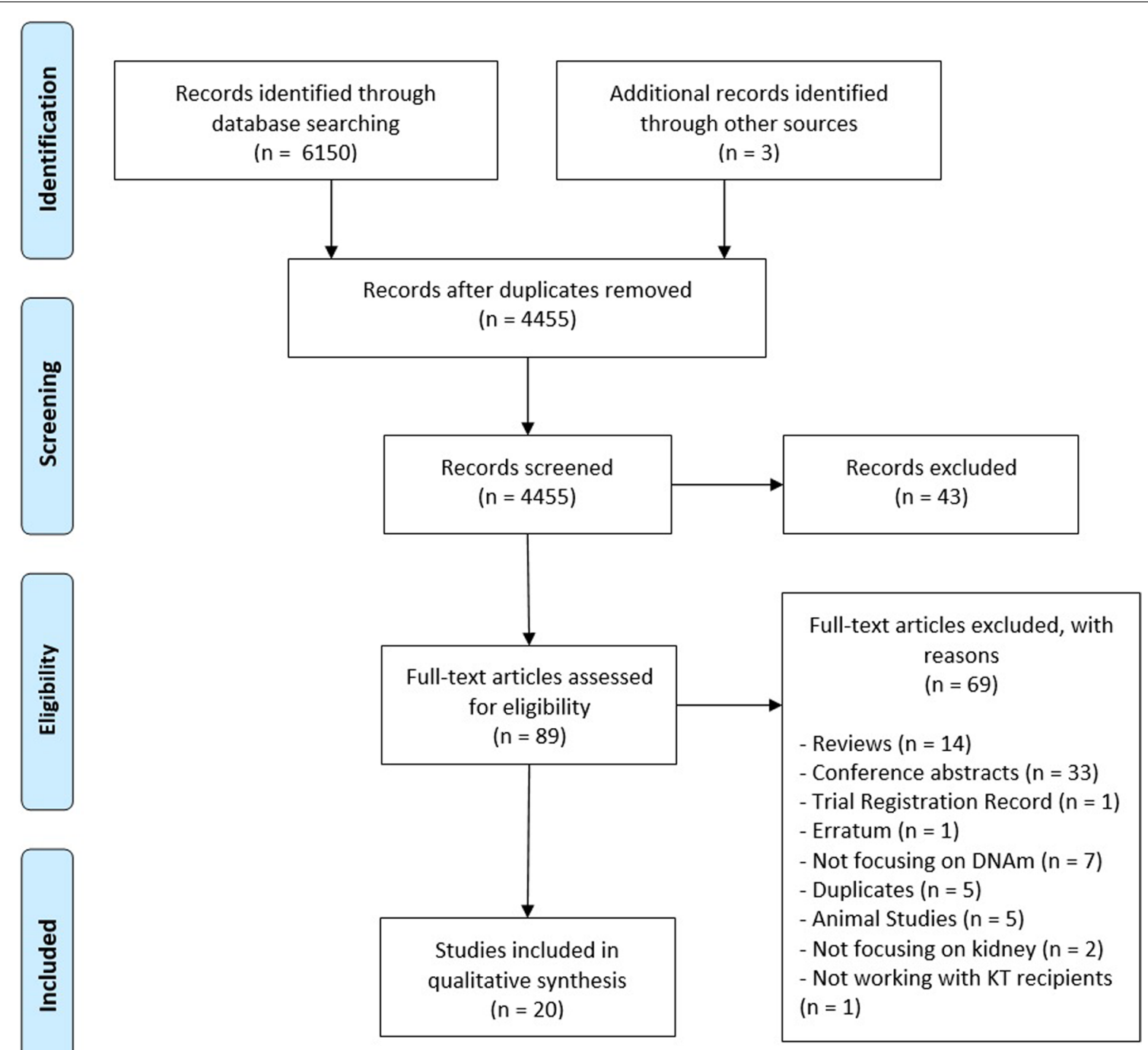

Fig. 1 Flow diagram of the systematic literature search 


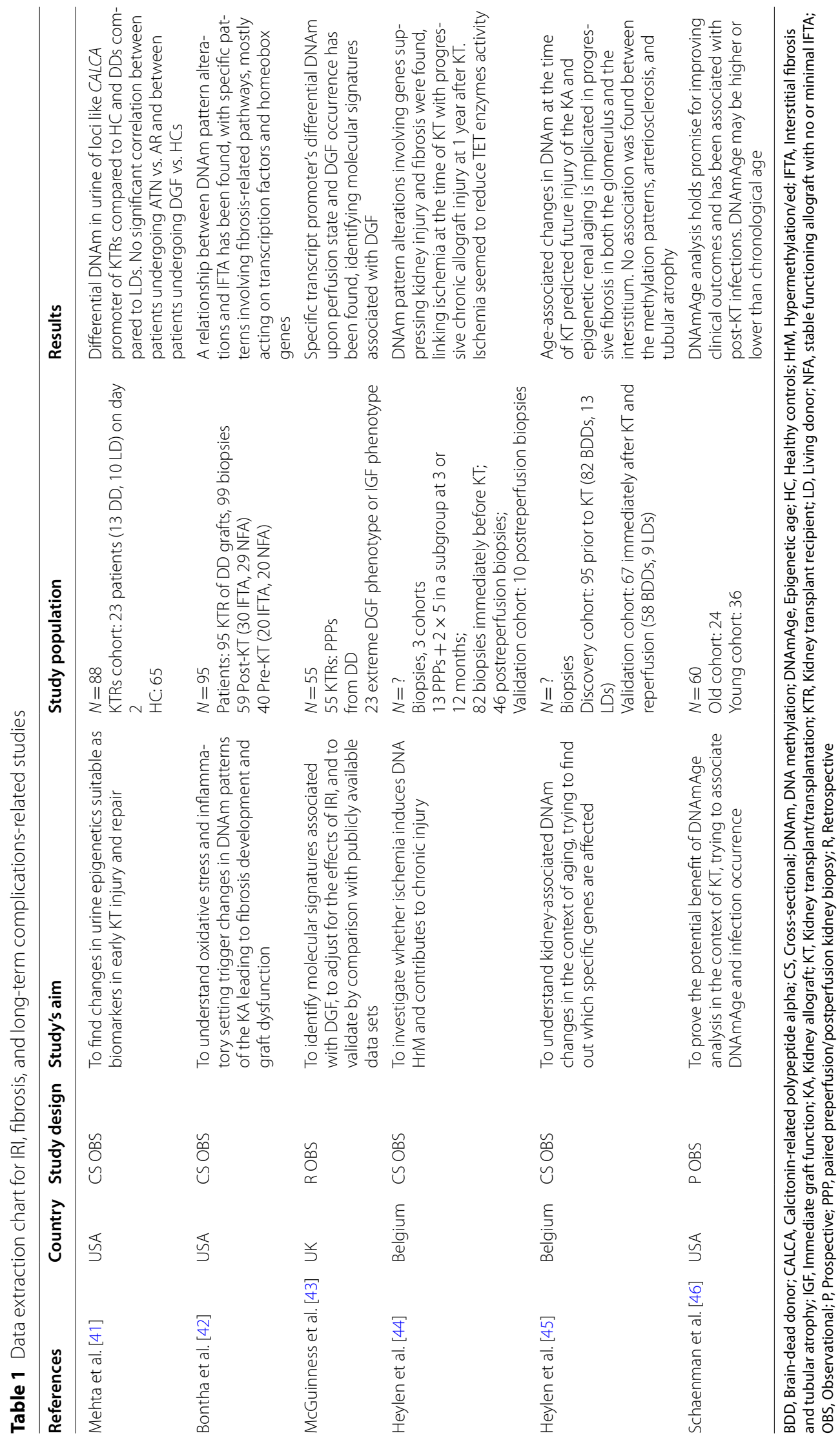




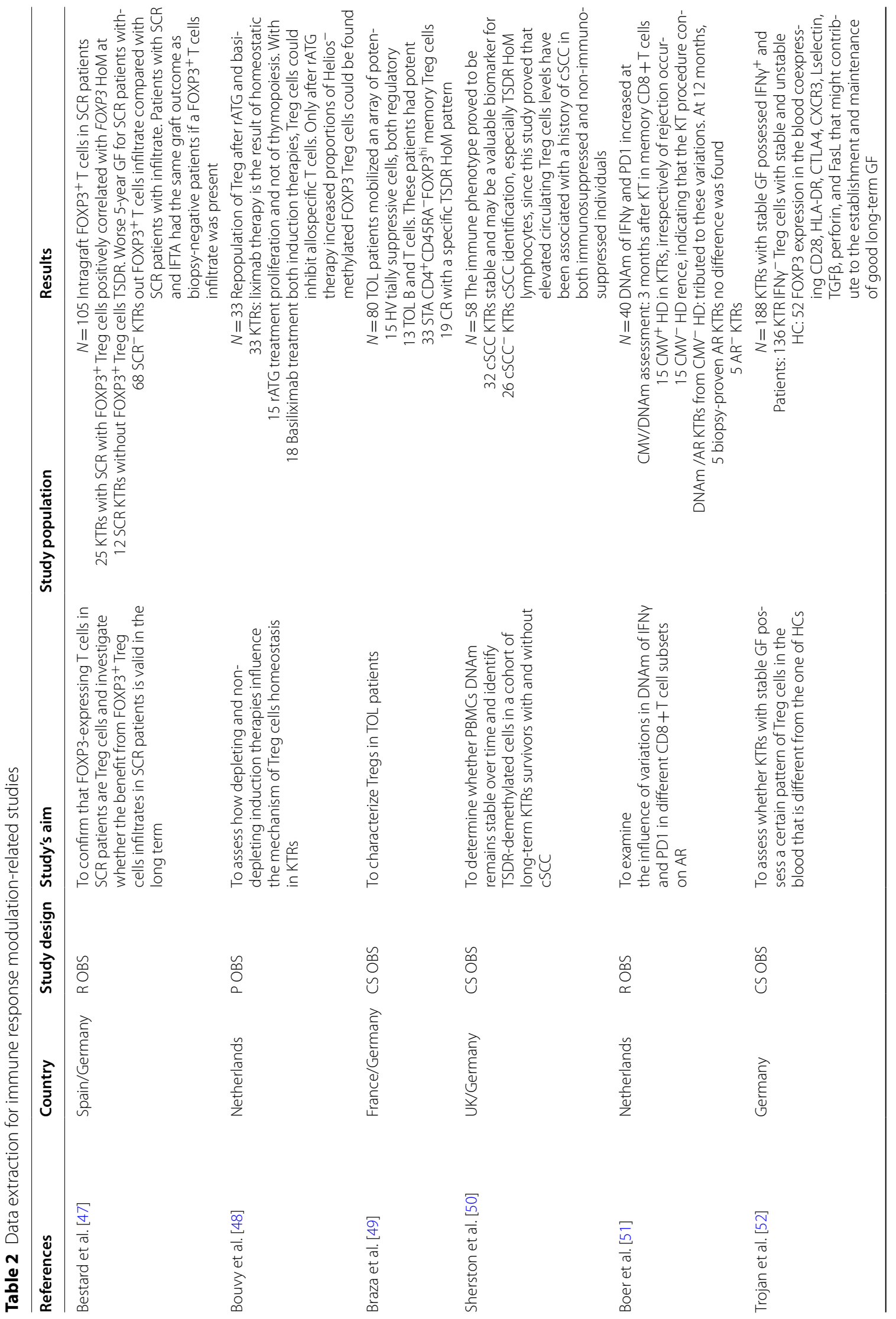




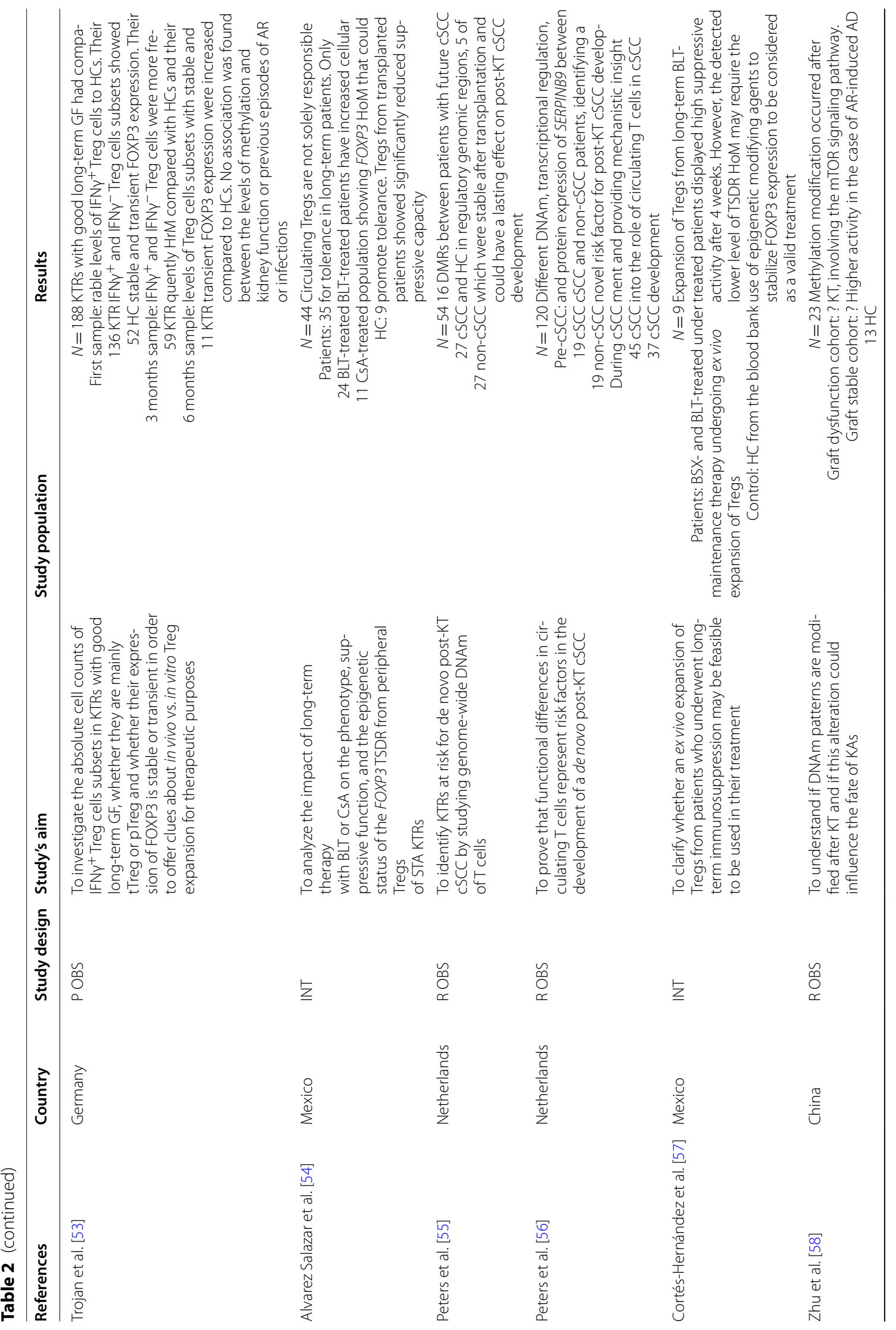




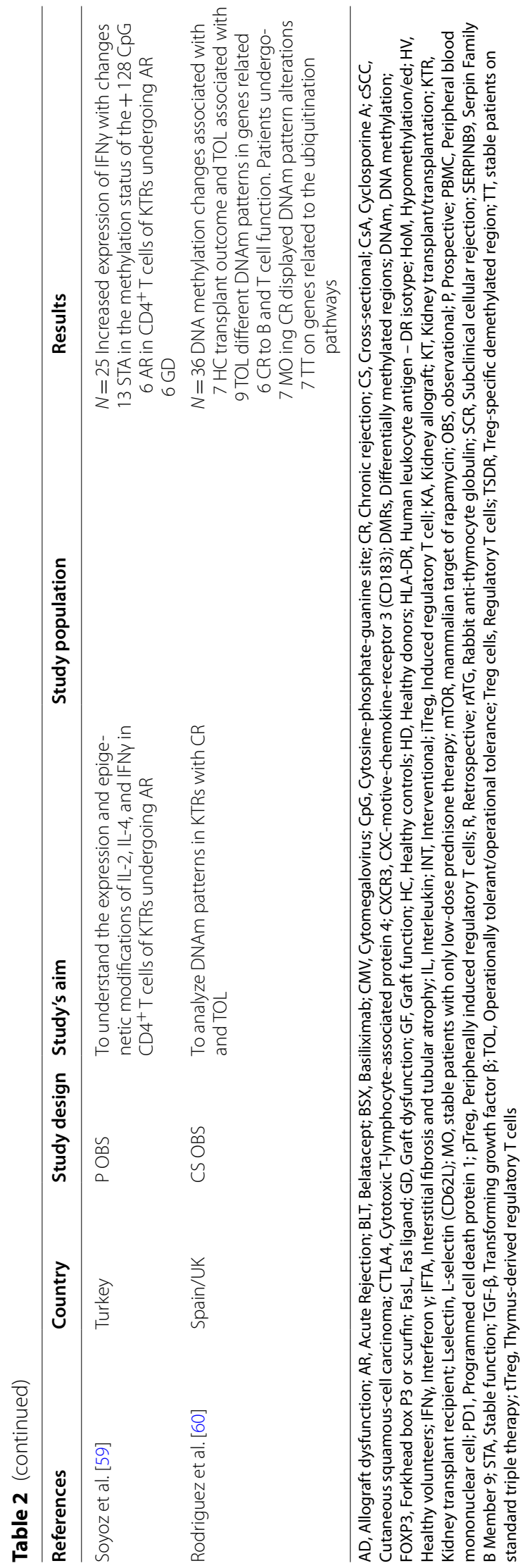




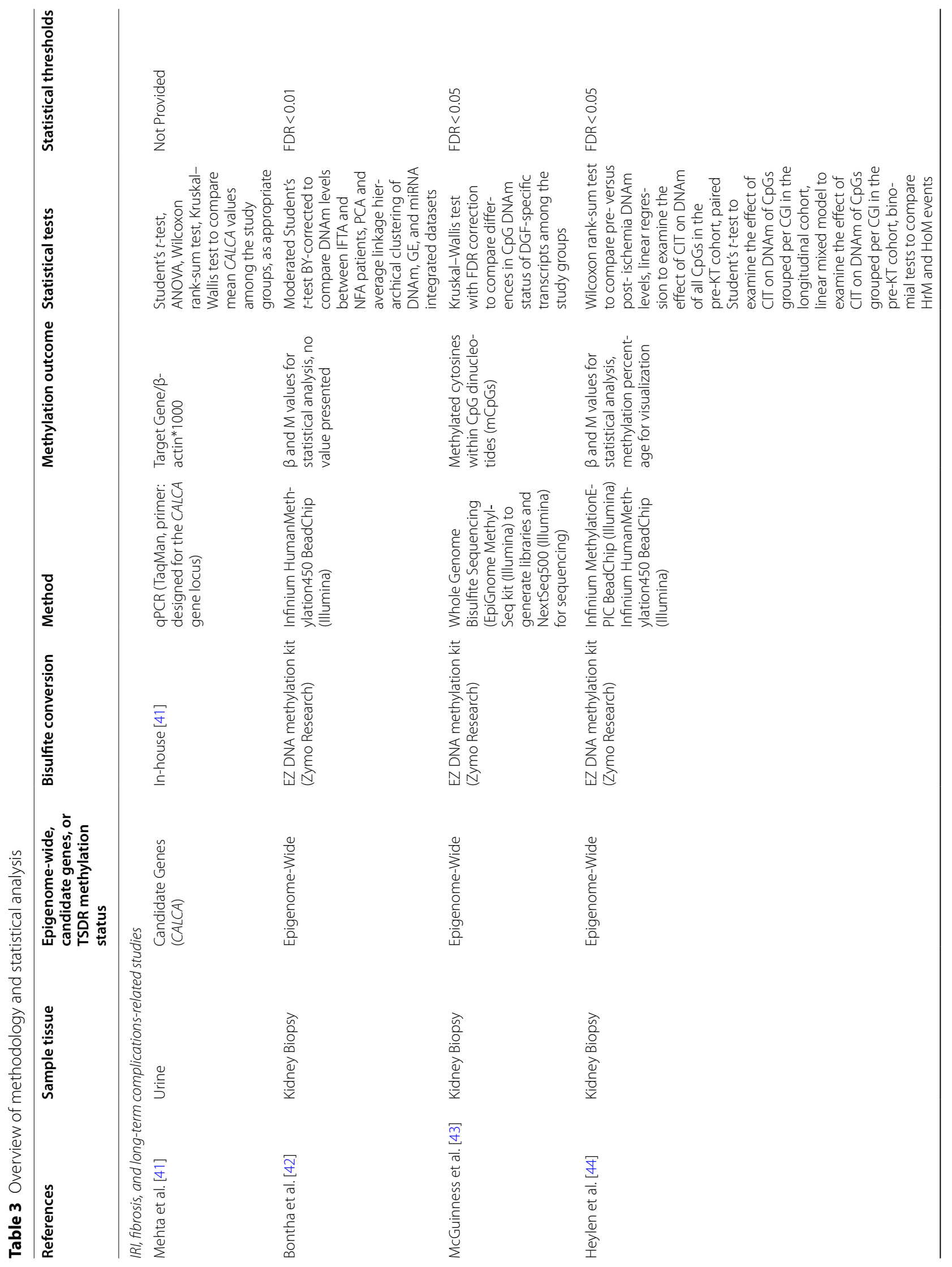




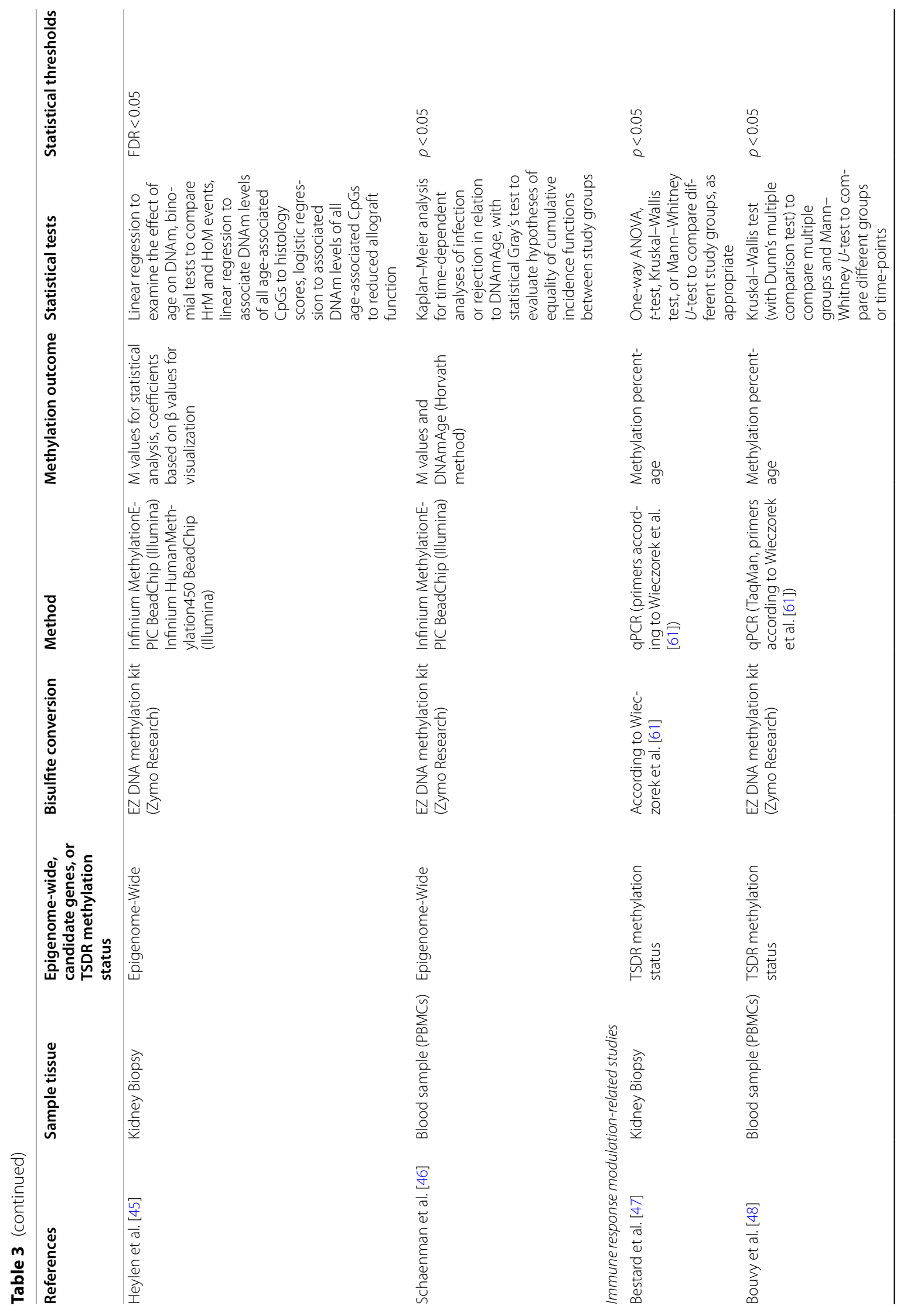




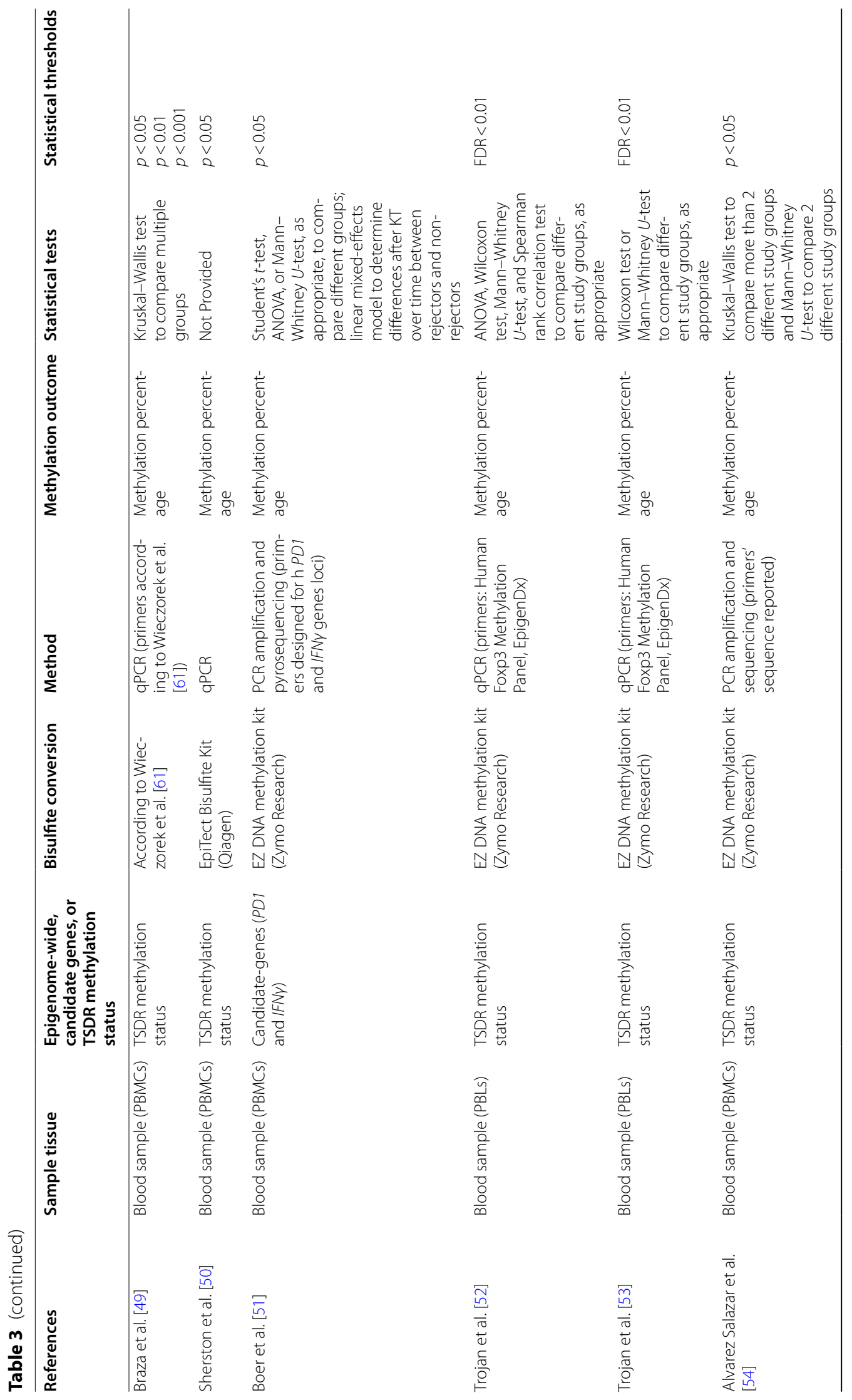




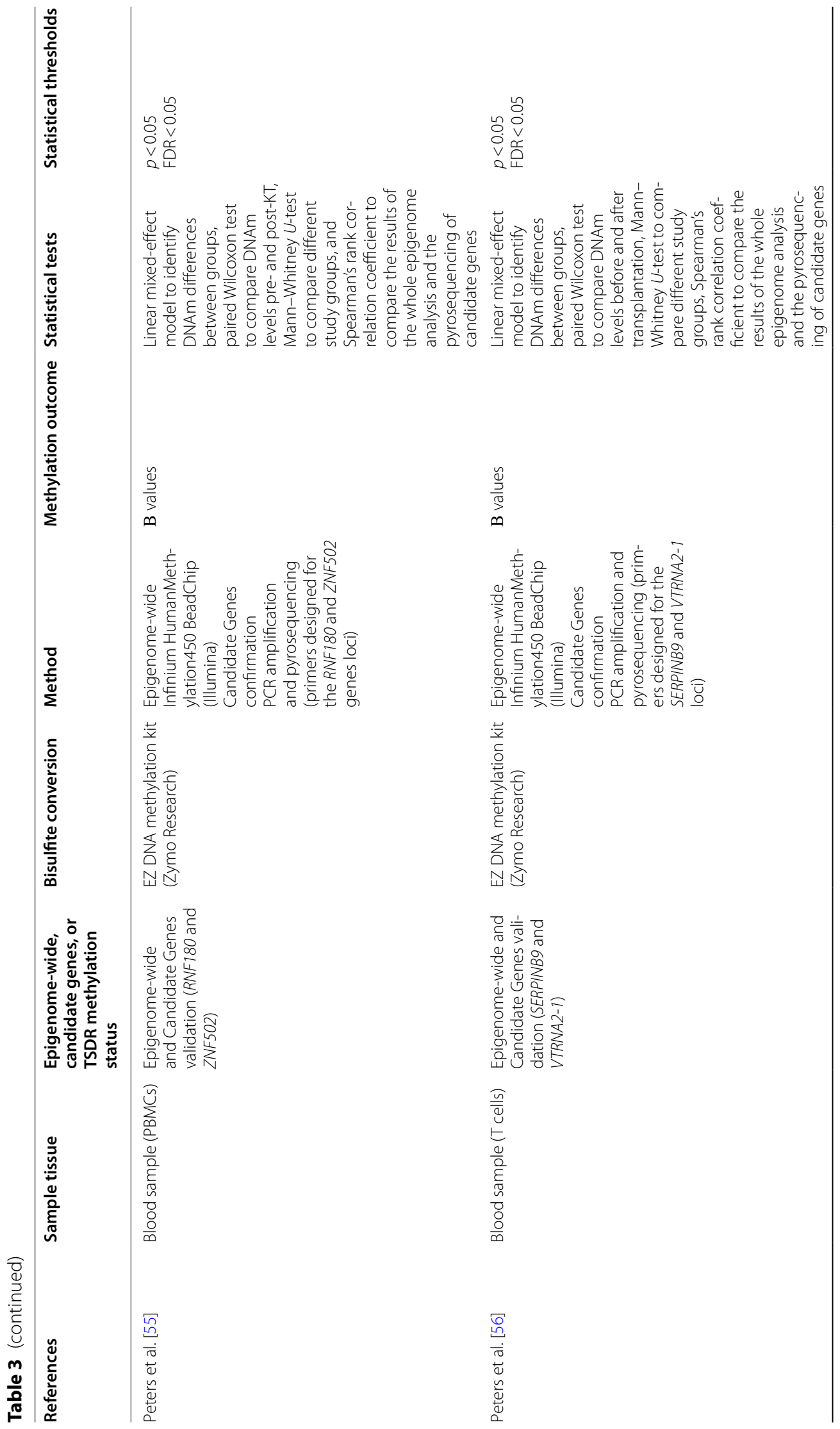




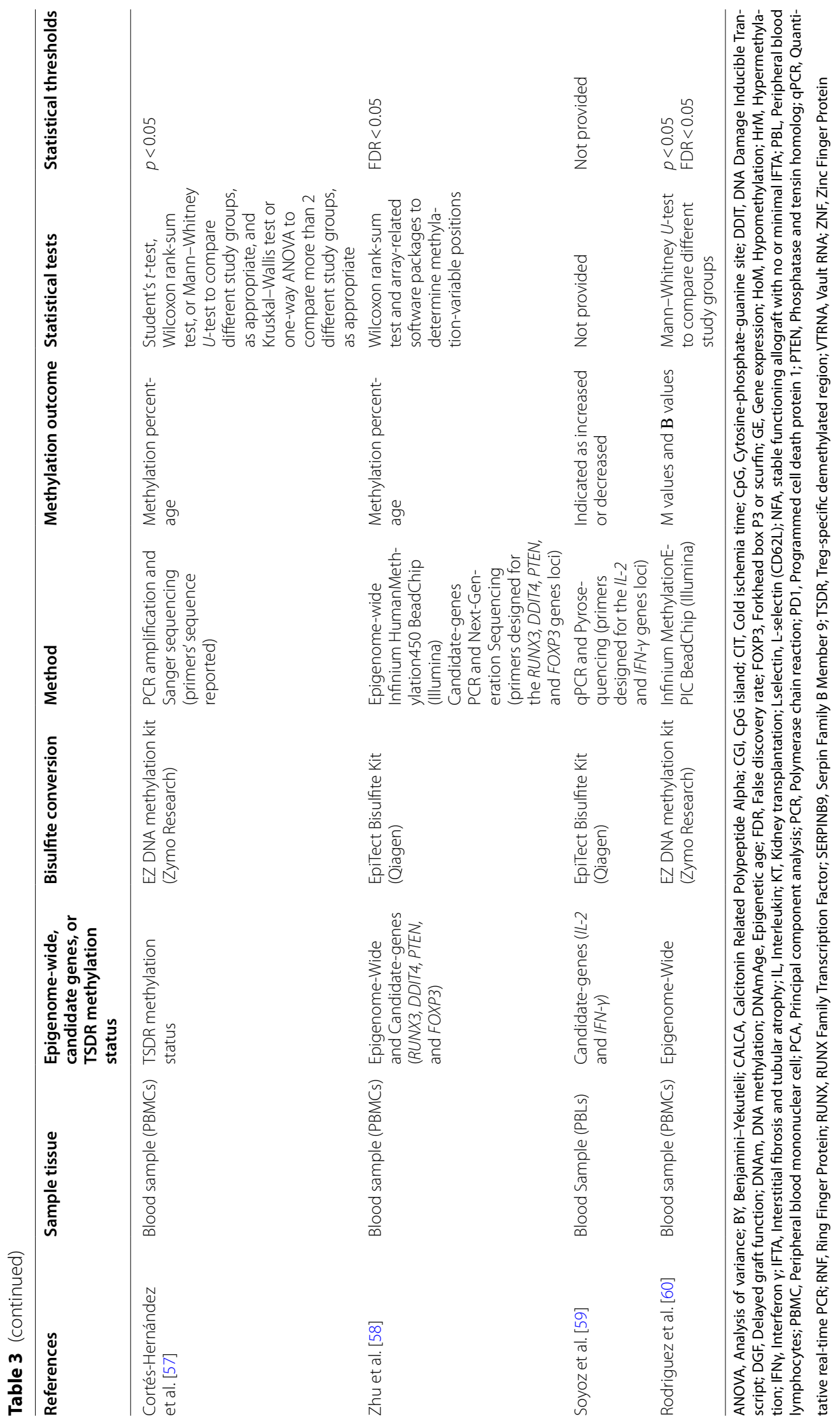


Treg-specific demethylated region (TSDR), while three studies performed epigenome-wide analysis as a first step and then investigated the methylation status of candidate genes.

\section{Ischemia-reperfusion injury, fibrosis, and long-term complications}

A total of six studies focused on IRI, fibrosis, and longterm complications; one study analyzed urine samples, four studies analyzed kidney biopsies, and one study analyzed blood samples. Detailed information on the individual studies is displayed in Tables 1 and 3. Briefly, aberrant hypermethylation of calcitonin-related polypeptide alpha $(C A L C A)$ gene in urine samples was significantly more likely to occur in kidney transplant recipients compared to healthy individuals [41]. Four studies investigated potential associations between the methylation state of different loci in kidney transplant biopsies and various clinical conditions that may occur at different time points after transplantation. In the first study, DNA methylation pattern alterations have been associated with early clinical conditions such as delayed graft function (DGF) [43]. The other three studies investigated DNA methylation in relation to ischemia, aging (evaluating DNAmAge), and fibrosis development to show relationships between these phenomena and the development of long-term complications [42, 44, 45]. The final more recent study investigated DNAmAge in peripheral blood mononuclear cells (PBMCs) samples and showed associations with posttransplant infection occurrence [46].

\section{Immune response modulation}

Fourteen studies reported on DNA methylation involved in immune-response modulation after kidney transplantation; one analyzed kidney biopsies, the other 13 focused on blood samples. Detailed information on the individual studies is displayed in Tables 2 and 3. Various $\mathrm{T}$ cell populations are involved in the allospecific immune response. Cytotoxic $\mathrm{CD} 8^{+} \mathrm{T}$ cells account for most of the adaptive immune response against the graft. Antigen-naïve $\mathrm{CD} 8^{+} \mathrm{T}$ cells are characterized by high methylation of effector genes, which are then demethylated after antigen recognition triggering effector genes expression and, consequently, the immune response activation. Among $\mathrm{CD} 4^{+} \mathrm{T}$ cells, regulatory $\mathrm{T}$ (Treg) cells are a subset of cells constitutively expressing high levels of the transcription factor FOXP3 along with the IL-2 receptor $\alpha$ chain CD25. These cells are crucial in the process of acceptance/tolerance of an allograft, considering their ability to suppress immune activation in an antigen-specific manner [62]. It is possible to identify stable Treg cells by measuring the methylation status of the TSDR as a demethylated TSDR characterizes
Treg cells, while this region is methylated in other cells. Among the included studies, eight studies investigated DNA methylation patterns in Treg cells associated with different clinical conditions related to kidney transplantation [51], such as subclinical rejection [47], tolerance $[49,60]$, stable graft function [52, 53], acute rejection $[58,59]$, and chronic rejection [60]. Three studies investigated the feasibility of Treg cells ex vivo expansion for therapeutic purposes in patients undergoing immunosuppressive treatment $[48,54,57]$. The final three studies investigated DNA methylation patterns associated with the development of cutaneous squamous-cell carcinoma (cSCC) $[50,55,56]$, a long-term complications of kidney transplantation that is related to the immunosuppressive regime that kidney transplant recipients must follow.

\section{Quality of evidence}

According to the Newcastle-Ottawa scale, the quality of all studies was considered moderate to good. The Newcastle-Ottawa scale assessment can be found in Additional files 2-4: Tables S2-S4. The overall Jadad score is good for randomized studies (Additional file 5: Table S5).

\section{Purpose of DNA methylation analysis in the included studies}

In Table 4, the included studies have been categorized into prediction, monitoring, and decision-making/intervention to assess for which purpose DNA methylation generally is studied.

\section{Discussion}

This systematic review on the application and value of DNA methylation as a biomarker in kidney transplantation shows heterogeneous and fragmented results. DNA methylation is a more accessible biomarker due to its low sensitivity to tissue handling compared with RNA or proteins and its analysis can even be performed on DNA isolated from small amounts of fixed tissue [63]. This biomarker could have an important role in different time points of the transplantation procedure and the subsequent short- and long-term follow-up. DNA methylation already proved, as other epigenetic mechanisms did, its role in the multiple biological events involved in posttransplant complications development $[64,65]$, with both the recipient and the donor organ continuously undergoing dynamic epigenetic modifications.

DNA methylation pattern alterations have already been associated in the past with IRI occurrence [23, 66-68]. The included studies showed how these alterations could be found in the urine of kidney transplant recipients [41] and could be related to perfusion state and DGF occurrence [43]. In the future, methylation assessment in 


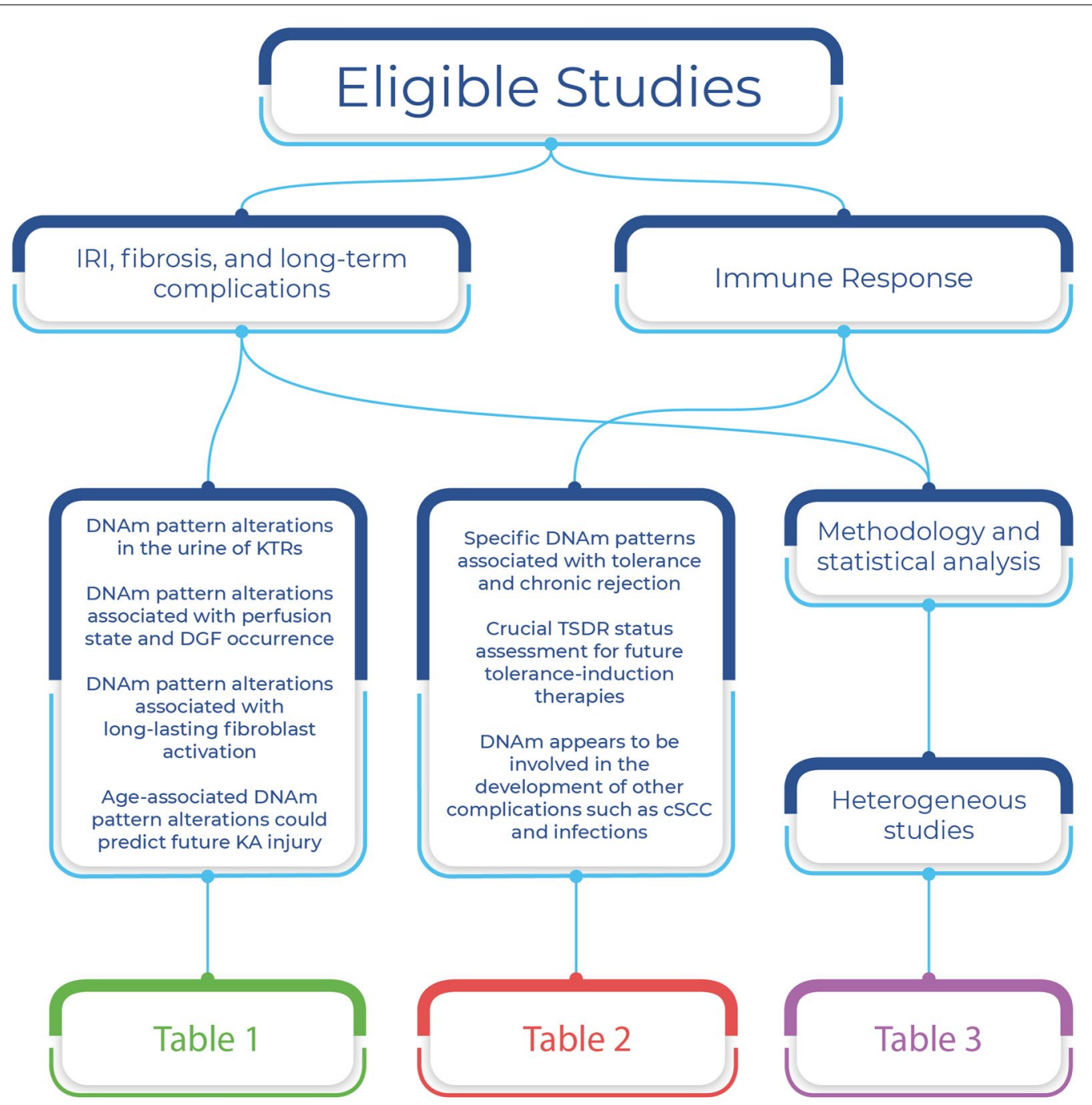

Fig. 2 Overview of the summarization strategy. Abbreviations: CSCC, Cutaneous squamous cell carcinoma; DGF, Delayed graft function; DNAm, DNA methylation; KA, Kidney allograft; KTR, Kidney transplant recipient; IRI, Ischemia-reperfusion injury; TSDR, Treg-specific demethylated region

different biological samples (kidney biopsy, urine, and blood) could be considered as an important early biomarker of acute kidney injury (AKI) during kidney transplantation, but further research is needed for a better understanding of its role in prediction, monitoring, and targeted therapy. Following the acute insult, fibrosis is the final common pathway of irreversible kidney transplant dysfunction. Its complex pathogenesis is triggered by an injury-induced fibroblast activation and matrix deposition that progresses even after the injury has disappeared. Evidence suggests that DNA methylation could in part be responsible for this process, acting through pro-fibrotic genes expression $[7,28,69]$. The included studies showed how DNA methylation pattern changes induced by oxidative stress and inflammatory setting could lead to this long-lasting fibroblast activation even in the context of kidney transplantation [42, 44] and how age-associated DNA methylation alterations at the time of the transplantation procedure could predict future injury [45]. Considering this, DNA methylation is a promising biomarker for the prediction of the development of chronic renal allograft dysfunction (CRAD) and could be used in the future for organ evaluation, prevention, and early intervention. As a consequence of this, preventing acute-injury-related DNA methylation alterations with modern preservation techniques could improve outcomes. The effect of normothermic machine perfusion on these biomarkers could be assessed and compared to that of static cold storage since this technique already proved to be able to recover previously discarded organs [70]. Moreover, these 
biomarkers could be used to optimize machine perfusion, acting on fluid compositions or operative parameters. Direct intervention on the markers through the perfusion fluid itself could also be an interesting opportunity in the future. The peculiar viable and isolated organ status typical of normothermic machine perfusion could allow the administration of demethylating agents and other additives while avoiding systemic side effects in the recipient. For a better understanding of the pathological processes leading to graft failure, and for an important role in monitoring and prediction of long-term complications, we recommend further investigations confirming the already established and promising associations, but also taking advantage of new high-resolution epigenome-wide DNA methylation assessment technologies to find new relevant loci and new associated patterns. The utilization of modern AI-based algorithms, for example, could help in integrating the massive amount of data provided by these new technologies to make accurate predictions, as will be described later.

DNA methylation could also have an important effect on long-term kidney function and development of fibrosis taking part in the recipient's immune system modulation. DNA methylation is involved in the regulation of activation pathways [71], in the determination of cell plasticity [2], as well as in the control of the transcriptional profiles and functions of memory $\mathrm{T}$ cells [72] and NK cells [73-75] and therefore could influence the strength of the allospecific immune response. Various $\mathrm{T}$ cell populations are involved in this response and the included studies showed how DNA methylation pattern alterations occurred after kidney transplantation in PBMCs [58] and different $\mathrm{T}$ cell types [51], highlighting a possible involvement of DNA methylation in these processes even in the context of kidney transplantation. A better understanding of these dynamics could be useful in the future for the evaluation of the recipient response against the graft. Among $\mathrm{CD} 4^{+} \mathrm{T}$ cells, Treg cells are a $\mathrm{CD} 4{ }^{+} \mathrm{CD} 25^{+} \mathrm{T}$ cell subset able to regulate inflammatory and immune responses [76, 77]. This subset was characterized by stable expression of FOXP3, a transcription factor that is essential for Treg cells function [78-80]. Stable expression of FOXP3 is obtained thanks to DNA demethylation of FOXP3 TSDR [81-84]. Treg cells can suppress the allograft-specific response using different mechanisms, ranging from suppressive cytokines to metabolite consumption [85]. Some of the included studies focused on the research of specific DNA methylation patterns associated with tolerance or other transplantrelated conditions in order to understand them and to be able to predict long-term complications $[47,49,52$, 60]. These studies show how DNA methylation assessment might be crucial for Treg cell characterization and
Table 4 Studies categorization based on DNA methylation main use

\begin{tabular}{ll}
\hline Category & References \\
\hline Prediction & {$[44-47,55,56,58]$} \\
Monitoring & {$[41-44,47-60]$} \\
Decision making/intervention & None \\
\hline
\end{tabular}

highlight the possible role of DNA methylation as a biomarker for post-transplant outcome prediction. A deeper understanding of the associations of specific DNA methylation patterns with post-transplant complications could also lead to the development of therapies based on epigenetic modifying agents.

Treg cells are central in the under-development tolerance-inducing cellular therapy. This therapy consists of the injection or implantation of living cells into a patient, with the potential to overcome the limitations of traditional drug treatment and to gain a deeper understanding of immune tolerance mechanisms [86]. Treg cell characterization through DNA methylation assessment is a crucial phase of these procedures, and TSDR methylation pattern has been studied in different kinds of patients to assess the suitability of these therapies. Kidney transplant recipients must undergo immunosuppression, a treatment that could impair Treg cell function as well as other immune cells function. For these reasons, the suitability of Treg cell-based therapy in transplant recipients has been studied by many researchers, focusing on the effect of different induction therapies [48] and different maintenance regimes [54, 57]. In one of the included studies, long-term treatment with belatacept showed better results in terms of the percentage of FOXP3 demethylated cellular populations compared to other maintenance therapies [54]. However, the same research group proved later that these expanded cellular populations may require the use of epigenetic modifying agents to stabilize the TSDR demethylated status [57]. Further investigations are needed to understand the potential effect of the most commonly used medications, such as tacrolimus, prednisone, and basiliximab. Direct intervention on TSDR methylation status already proved to stabilize Treg cells for adoptive cell therapy $[87,88]$, but further studies are needed to clarify this aspect in the context of kidney transplantation and to improve current isolation and expansion techniques. Tolerance induction through Treg cells administration appears to be one of the most promising research topics trying to solve the organ deficiency problem and TSDR demethylation status assessment could be crucial for the characterization, ex vivo expansion, and stabilization of allospecific autologous Treg cell populations. 
For the property of influencing and directing the immune response, DNA methylation appears also to be involved in the development of other complications than graft failure. Several studies focused on DNA methylation's predictive value for complications such as cSCC $[50,55,56]$ and infections [46], underlining the possible role of DNA methylation as a biomarker for other complications development and prediction. Although promising candidates with prognostic values significantly associated with survival and complications occurrence have already been identified, none of them is ready to be transferred into clinical practice because of the high heterogeneity of the studies [89].

Research in the field of kidney transplantation should be more focused on the predictive feature of DNA methylation modifications. Identifying patients at high risk for rejection or long-term complications through DNA methylation assessment would be a suited tool to guide clinical decision-making. As shown in Table 4, most of the research groups focused on prediction and monitoring, while currently, not a single study used DNA methylation for intervention or decision making. With the progress of tolerance induction therapy research, this could change, giving DNA methylation a central role in direct intervention and therapeutic strategies.

The growing interest for less invasive procedures to detect organ damage, the so-called liquid biopsies, raised the interest of the research community for DNA methylation analysis to quantify cell-free DNA (cfDNA). DNA methylation assessment is crucial for cfDNA origin identification [90]. After solid organ transplantation, donor-derived cfDNA (ddcfDNA) is released into the circulation, and the amount of ddcfDNA is representative of graft integrity. Dd-cfDNA can be distinguished from cfDNA originating from the recipient thanks to the genomic differences between donor and recipient typical of organ transplantation [91]. Nevertheless, tissue-specific DNA methylation patterns of cfDNA also provide the opportunity to identify the tissue origin of the detected genetic material [92]. An increase in ddcfDNA in blood plasma, either detected based on genomic differences or tissue-specific methylation patterns [93], has been reported to identify acute rejection $[94,95]$. Moreover, methylated cfDNA in urine is one of the markers included in the Kidney Injury Test (KIT) to diagnose kidney injury as well as kidney allograft rejection $[96,97]$.

The problem of the heterogeneity of the studies concerning DNA methylation as a biomarker for kidney transplantation should be addressed. Designs of the included studies were mainly retrospective and covered mostly empirical evidence from case series. In consequence, the patient populations were also heterogeneous with a large variation in assessed epigenetic modifications, outcomes, and study designs. For what concerns phenotypes, more standardized definitions should be adopted. Allograft rejection, CRAD, IRI, kidney fibrosis, and DGF, for instance, are all phenotypes that can be defined with slight differences that can prevent comparison in a statistically valid meta-analysis. Despite common phenotype definitions, studies can also differ in other characteristics of the analyzed populations. For example, different duration of end-stage renal disease, time points of DNA methylation analysis, and purposes for DNA methylation analysis, albeit of potential scientific interest, are hampering the comparability of different studies in this early research phase. Analog considerations can be made for primary endpoints, with little agreement between study groups and the adoption of outcomes that might drastically complicate logistics, such as graft survival or recipient survival [98]. Moreover, the included studies adopted different DNA methylation analysis strategies, for example, different experimental approaches such as gene-specific or genome-wide analyses (summarized in Table 3) and even within similar experimental approaches, different technologies for DNA methylation analysis have been adopted. Furthermore, almost none of the studies report validation of their findings with another technique like pyrosequencing or biological validation as in variation in mRNA or protein expression.

Overall, the high number of confounders and the variety of arrays and protocols that have been used to assess common DNA methylation patterns prevent these studies from producing common statistically valid knowledge. An international clear consensus among the active research groups in this field should be discussed to designate important endpoints and produce more comparable results that could stimulate further research and generate new knowledge through the use of scientifically valid meta-analyses. Future studies should try to adopt prospective designs, with DNA methylation assessments performed before kidney transplantation and at specific time points after the procedure. Specific endpoints, DNA methylation analysis, and biological validation protocols should be adopted by different groups. This will not only enhance the comparability of these studies but will also lead to more cost-effective research. Here in this review, we provide insight on some of the previously discussed heterogeneities and on other potential implementations to help create a discussion that one day, hopefully, might lead to the development of DNA-methylation-based clinical tools to support decision-making in the kidney transplantation field.

In the last ten years, technology in the field of DNA methylation assessment quickly advanced, supported by the exponential growth of computational techniques 
for big data analysis. DNA methylation profiling techniques can be grouped based on the properties that are used to discriminate between methylated and unmethylated sites, namely enzyme digestion, affinity enrichment, and bisulfite conversion [99]. Due to their low resolution and to the quick development of bisulfite-conversionbased assays, enzyme-based (i.e., comprehensive highthroughput arrays for relative methylation, CHARM [100]) and affinity-based assays (i.e., methylated DNA immunoprecipitation, MeDIP [101]) are scarcely adopted in recent times and therefore will not be discussed in this manuscript [99]. In bisulfite-conversion-based methods, methylation-dependent changes are generated as bisulfite deaminates unmethylated cytosines into uracils, while methylated cytosines remain unchanged. These techniques, including methylation arrays, whole-genome bisulfite sequencing (WGBS), and reduced-representation bisulfite sequencing (RRBS), are characterized by single-base resolution and are among the most commonly used assays to study genome-wide methylation. Among methylation arrays, Illumina's Infinium HumanMethylation450 BeadChip ${ }^{\circledR}(450 \mathrm{~K}$ array $)$ and the updated version Illumina's Methylation EPIC BeadChip ${ }^{\circledR}$ (EPIC array) are the most commonly used technologies to investigate the whole methylome [102]. These assays combine bisulfite conversion with amplification and hybridization of the converted DNA to arrays with predefined probes to assess the methylation status of around 450,000 and 850,000 methylation sites, respectively [103]. These technologies are characterized by high cost-effectiveness and by the need for low amounts of input DNA. However, their coverage is dependent on the array design, i.e., the selection of the predefined probes [99]. In WGBS, DNA is fragmented through sonication, repaired, added of an adenine base on the 3' end, and successively ligated to methylated adapters. After size selection, bisulfite conversion is applied and the resulting genetic material is amplified and sequenced [104]. The great advantage of this technique is its ability to evaluate the methylation state of almost every CpG site in the genome. However, WGBS is expensive and impaired by DNA degradation after bisulfite treatment [99]. RRBS integrates restriction enzyme digestion, bisulfite conversion, and nextgeneration sequencing to analyze only specific fragments covering more than $85 \%$ of CpG islands while decreasing cost [105]. Nevertheless, RRBS focuses on promoters and areas close to the restriction site with low coverage of intragenic and distal regulatory elements [99]. Heterogeneous experimental approaches have been applied in the selected studies, introducing multiple confounders that impair comparability (summarized in Table 3). For instance, nine studies included in this review investigated DNA methylation on a whole-epigenome-scale all using bisulfite-conversion-based techniques, but the efficiency of bisulfite conversion was mostly not examined, while it represents a potential confounder. Methylation array was the most represented technique, accounting for eight out of nine studies, while only one study adopted WGBS. Considering the low cost and the need to increase the amount of available comparable data, we believe that the use of methylation arrays might be ideal in this early phase of research. Among methylation arrays, the $450 \mathrm{~K}$ array was the most common assay adopted in the studies included in this review while its updated version, the EPIC array, is being adopted by more and more research groups in recent times. The EPIC array contains over 850,000 probes, covering more than $90 \%$ of the probes of the $450 \mathrm{~K}$ array, but also covering sites in distal regulatory regions such as enhancer regions, overcoming one of the $450 \mathrm{~K}$ array's main limitations [106]. Considering the better performances, the low price, and the compatibility with the $450 \mathrm{~K}$ array data shown by the very high persample correlation between the results of the two assays performed over the same samples [107], we recommend the use of the EPIC array for epigenome-wide DNA methylation assessment to take advantage of this solid technology as a research community, to produce comparable results, and to accelerate the process that might bring DNA methylation assessment into clinical practice.

After DNA methylation analysis, appropriate data analysis pipelines should be adopted to optimize the quality of the collected data. For instance, Wang et al. proposed an analysis framework for data collected with the $450 \mathrm{~K}$ arrays that, with the appropriate adaptations, is valid for the updated EPIC array [108]. Moreover, epigenetic data seems to be optimally suitable for machine learning, thanks to the stability over time of epigenetic modifications [109] and to the increasing availability of large-scale repositories [110-112]. As reviewed by Rauschert et al., different machine learning methods have been applied to epigenomic datasets for the development of diagnostic systems, frequently using supervised learning methods [113]. Even though in the above-mentioned review the most adopted algorithm was random forest, we believe that deep learning algorithms [114] might have a big impact in the discovery of clinically useful epigenetic biomarkers for kidney transplantation thanks to the currently available high computational power and their capability to process highly dimensional datasets and identify complex patterns. However, we believe that a coordinated effort from the research community to make large epigenetic datasets publicly available and to elaborate standardized preprocessing pipelines is needed. These pipelines should include standardized normalization and imputation methods to increase data compatibility. These efforts would be crucial for a successful development and 


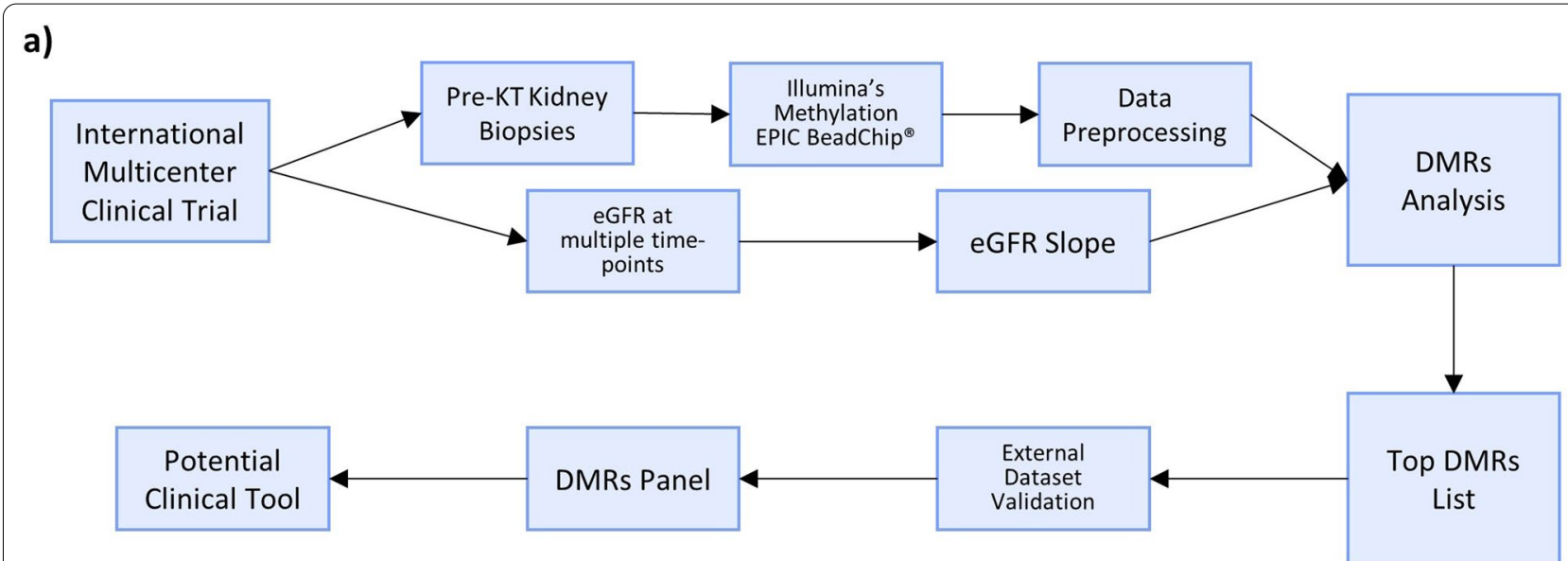

b)

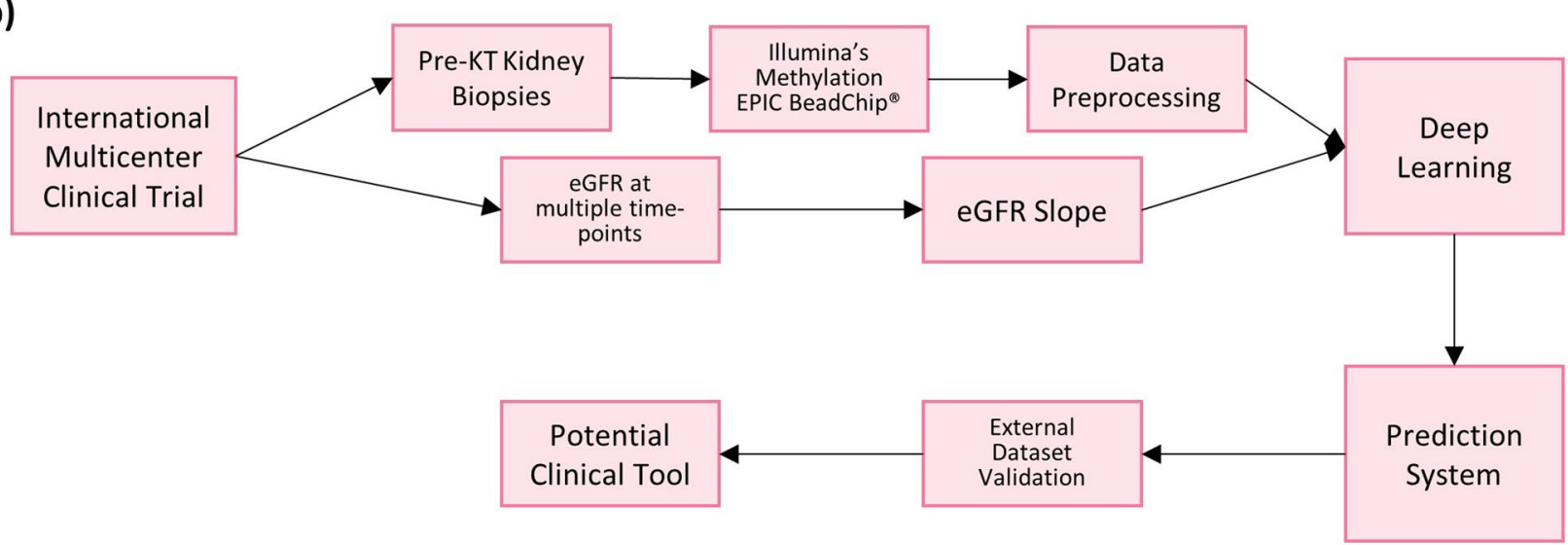

Fig. 3 Overview of the proposed biomarker discovery pipelines. a Workflow of a proposed DNA methylation analysis pipeline for the discovery of differentially methylated regions associated with eGFR slope for the development of a limited panel that could be the base of a potential clinical tool. $\mathbf{b}$ Workflow of a proposed DNA methylation analysis pipeline for the development of a deep-learning-based prediction system. Abbreviations: DMR: Differentially methylated regions; eGFR: Estimated glomerular filtration rate; KT: Kidney transplantation

implementation of deep-learning-based tools in a clinical context.

An ideal study setting for the discovery of clinically relevant biomarkers should collect data from a purposely designed multicenter international clinical trial. Since different DNA methylation patterns of multiple CpG sites between people of different ethnicity have been reported in the past [115], a high number of participants of different ethnicities and from different international centers would improve generalizability and tackle overfitting, one of the most common issues that accompany modern algorithms. In this early phase, analyzing pre-transplant kidney biopsies would be convenient considering the less invasive approach that minimizes the risk for complications. DNA methylation analysis should be performed on an epigenome-wide scale in order to identify new biomarkers that might associate with the outcome of interest. We believe that at this stage the research community should support the use of methylation arrays, i.e., the EPIC array, in order to produce comparable data with an accurate, widely available, and relatively cheap technology. Bisulfite conversion is a potential confounder, and its efficiency should be reported and taken into account during data analysis. For what concerns the investigated outcome, traditional clinical biomarkers of transplant outcome such as graft survival or recipient survival are characterized by low rates, requiring problematic sample sizes and follow-up periods. For this reason, the use of surrogate and composite endpoints, such as the estimated glomerular filtration rate (eGFR) slope, might improve data quality and speed up the implementation of DNAmethylation-based biomarkers into clinical practice [98, 116]. Data preprocessing should be handled in a standardized way, paying particular attention to quality control and within-array normalization. For this purpose, R [117] packages such as Minfi [112] and methylumi [118] might 
be the most suitable solution. Since the EPIC array uses two different types of probes, correction of probe design bias is also of uttermost importance. Multiple strategies have been elaborated to tackle this issue and solutions like Beta Mixture Quantile normalization (BMIQ) [119] or Regression on Correlated Probes (RCP) [120] should be appropriate. Two different approaches might then be followed (summarized in Fig. 3). A first approach (Fig. 3a) would be to use the normalized data to identify differentially methylated probes/differentially methylated regions using R packages like Minfi. Afterward, biological interpretation and gene ontology term enrichment analysis might then be performed with the R package missMethyl [121]. The detected differentially methylated regions might then be considered as candidate biomarkers for the selected outcome, and their association might be validated on other datasets. Validating these biomarkers might be of great value not only for monitoring and prognosis but also to better understand the processes underlying unfavorable post-transplant outcomes to elaborate novel therapeutic strategies. Successively, a panel composed of the eventually discovered biomarkers might be elaborated, leading to the development of easier and cheaper DNA methylation analysis pipelines of candidate genes based on bisulfite pyrosequencing that could enter the clinical practice. A second approach (Fig. 3b) would be based on the use of deep learning to produce surrogate endpoint predictions. Unfortunately, the most important features for these predictions would not be known, making the use of the whole EPIC array necessary even for the eventually developed clinical tool. However, the characteristics of epigenomic data, with the high number of probes and the multiple relationships between them, make it ideal to exploit the potential of deep learning to produce accurate predictions, making a big step toward the development of a clinical tool to support decision making. For that purpose, the use of the novel deep learning method MethylNet would be ideal to handle this type of data, considering its ability to construct embeddings, make predictions, and capture nonlinear interactions [122].

\section{Conclusions}

DNA methylation is involved in acute ischemic injury, CRAD, and immune response modulation. So far, studies included in this review are heterogeneous in study design, DNA methylation analysis protocol, biological samples, and outcomes. DNA methylation analysis is increasingly being used in the field of kidney transplantation, but it is too early to affirm DNA methylation as a clinically relevant biomarker important for prevention, monitoring, and intervention. The studies described in this review highlighted its potential, especially considering the newest epigenome-wide methylation assessment technologies and novel discoveries in the field of big data analysis. An international agreement on study settings is needed to stimulate further research and achieve the first milestones in the quest for clinically useful biomarkers.

\begin{abstract}
Abbreviations
450 K array: Illumina's Infinium HumanMethylation450 BeadChip ${ }^{\circledR}$; AKI: Acute kidney injury; BMIQ: Beta-mixture quantile normalization; CALCA: Calcitoninrelated polypeptide alpha; CD: Cluster of differentiation; cfDNA: Cell-free DNA; CHARM: Comprehensive high-throughput arrays for relative methylation; CpG: Cytosine-phosphate-guanine; cfDNA: Cell-free DNA; CRAD: Chronic renal allograft dysfunction; CSCC: Cutaneous squamous-cell carcinoma; ddcfDNA: Donor-derived cell-free DNA; DGF: Delayed graft function; DNAmAge: Epigenetic age; eGFR: Estimated glomerular filtration rate; EPIC array: Illumina's Methylation EPIC BeadChip ${ }^{\circledR}$; FOXP3: Forkhead box P3 or scurfin; HLA: Human leukocyte antigen; IL: Interleukin; IRI: Ischemia-reperfusion injury; KIT: Kidney injury test; MeDIP: Methylated DNA immunoprecipitation; PBMCs: Peripheral blood mononuclear cells; RCP: Regression on correlated probes; RRBS: Reduced-representation bisulfite sequencing; Treg: Regulatory T;TSDR: Tregspecific demethylated region; WGBS: Whole-genome bisulfite sequencing.
\end{abstract}

\section{Supplementary Information}

The online version contains supplementary material available at https://doi. org/10.1186/s13148-022-01241-7.

Additional file 1: Table S1. Description of data: Search terms used for this systematic review.

Additional file 2: Table S2. Description of data: Risk of Bias assessment with the Newcastle-Ottawa scale for case-control studies.

Additional file 3: Table S3. Description of data: Risk of Bias assessment with the Newcastle-Ottawa scale for cohort studies.

Additional file 4: Table S4. Description of data: Risk of Bias assessment with the Newcastle-Ottawa scale for cross-sectional studies.

Additional file 5: Table S5. Description of data: Risk of Bias assessment with the Jadad scale for clinical trials.

\section{Acknowledgements}

The authors would like to thank the 'Consorzio per la Ricerca Sanitaria' (CORIS) of the Veneto Region, Italy (L.i.f.e.L.a.b. Program) for the support. The authors would like to thank Wichor Bramer, Sabrina Meertens-Gunput, and Maarten F.M. Engel for their assistance with the literature search. The authors would like to thank Federico Maria Campisi for his work on the graphics of Figure 2.

\section{Authors' contributions}

IC and RCM designed and supervised the study. IC, TAG, and RCM performed the systematic literature search. IC and TAG extracted data from eligible studies. IC, TAG, and RCM evaluated risk of bias for the included studies. IC drafted the manuscript. KB supported and supervised the writing of paragraphs concerning liquid biopsies and DNA methylation assays. MvB supported the writing of paragraphs concerning the use of modern bioinformatics tools. LF, SP, FN, MC, MvB, KB, HJANK, MC-VG, RCM, and IC critically reviewed the manuscript and approved the final draft. All authors read and approved the final manuscript.

\section{Affiliations}

L.i.f.e.L.a.b. Program, Consorzio per la Ricerca Sanitaria (CORIS), Veneto Region, Via N. Giustiniani 2, 35128 Padova, Italy.

\section{Funding}

This research did not receive any specific grant from funding agencies in the public, commercial, or not-for-profit sectors. 
Availability of data and materials

Not applicable.

\section{Declarations}

Ethics approval and consent to participate

Not applicable.

\section{Consent for publication}

Not applicable.

\section{Competing interests}

The authors declare that they have no competing interests.

\section{Author details}

${ }^{1}$ Division of HPB and Transplant Surgery, Department of Surgery, Erasmus MC, University Medical Center Rotterdam, Doctor Molewaterplein 40, 3015GD Rotterdam, the Netherlands. ${ }^{2}$ Department of Pathology and Clinical Bioinformatics, Erasmus MC, University Medical Center Rotterdam, Doctor Molewaterplein 40, 3015GD Rotterdam, the Netherlands. ${ }^{3}$ Erasmus MC Transplant Institute, Erasmus MC, University Medical Center Rotterdam, Doctor Molewaterplein 40, 3015GD Rotterdam, the Netherlands. ${ }^{4}$ Kidney and Pancreas Transplantation Unit, Department of Surgical, Oncological and Gastroenterological Sciences, Padua University Hospital, Via Giustiniani 2, 35128 Padua, Italy. ${ }^{5}$ Occupational Medicine, Department of Cardiac, Thoracic, Vascular Sciences and Public Health, Padua University, Via Giustiniani 2, 35128 Padua, Italy. ${ }^{6}$ Environmental and Respiratory Physiology Laboratory, Department of Biomedical Sciences, Padua University, Via Marzolo 3, 35131 Padua, Italy. ${ }^{7}$ Institute of Anaesthesia and Intensive Care, Department of Medicine - DIMED, Padua University Hospital, Via Cesare Battisti 267, 35128 Padua, Italy. ${ }^{8}$ Division of Nephrology and Transplantation, Department of Internal Medicine, Erasmus MC, University Medical Center Rotterdam, Doctor Molewaterplein 40, 3015GD Rotterdam, The Netherlands. ${ }^{9}$ Institute of Experimental Medicine and Systems Biology, RWTH Aachen University, Pauwelsstraße 30, 52074 Aachen, Germany.

Received: 13 October 2021 Accepted: 27 January 2022

Published online: 07 February 2022

\section{References}

1. Wolfe RA, et al. Comparison of mortality in all patients on dialysis, patients on dialysis awaiting transplantation, and recipients of a first cadaveric transplant. N Engl J Med. 1999;341(23):1725-30.

2. Mas VR, Le TH, Maluf DG. Epigenetics in kidney transplantation: Current evidence, predictions, and future research directions. Transplantation. 2016;100(1):23-38.

3. Moreso F, Hernandez D. Has the survival of the graft improved after renal transplantation in the era of modern immunosuppression? Nefrología (English Edition). 2013;33(1):14-26.

4. Dupont PJ, Manuel O, Pascual M. Infection and chronic allograft dysfunction. Kidney Int Suppl. 2010;78(119):S47-53.

5. Campistol JM, et al. Chronic allograft nephropathy: a clinical syndrome: early detection and the potential role of proliferation signal inhibitors. Clin Transplant. 2009;23(6):769-77.

6. Nankivell BJ, Kuypers DR. Diagnosis and prevention of chronic kidney allograft loss. Lancet. 2011:378(9800):1428-37.

7. Agodi A, et al. Unveiling the role of DNA methylation in kidney transplantation: novel perspectives toward biomarker identification. BioMed Res Int. 2019;2019:1602539.

8. Matas AJ, et al. OPTN/SRTR 2012 annual data report: kidney. Am J Transplant. 2014;14(Suppl 1):11-44

9. Daniëls $L$, et al. The clinical significance of epitope mismatch load in kidney transplantation: a multicentre study. Transpl Immunol. 2018;50:55-9.

10. Lefaucheur $\mathrm{C}$, et al. Preexisting donor-specific HLA antibodies predict outcome in kidney transplantation. J Am Soc Nephrol. 2010;21(8):1398-406.

11. Safa K, Magee CN, Azzi J. A critical review of biomarkers in kidney transplantation. Curr Opin Nephrol Hypertens. 2017;26(6):509-15.
12. Menon MC, Murphy B, Heeger PS. Moving biomarkers toward clinical implementation in kidney transplantation. J Am Soc Nephrol. 2017:28(3):735-47.

13. Cravedi P, Heeger PS. Immunologic monitoring in transplantation revisited. Curr Opin Organ Transplant. 2012;17(1):26-32.

14. Portela A, Esteller M. Epigenetic modifications and human disease. Nat Biotechnol. 2010;28(10):1057-68.

15. Esteller M. Epigenetics in evolution and disease. Lancet. 2008:372:590-6.

16. Straussman $\mathrm{R}$, et al. Developmental programming of $\mathrm{CpG}$ island methylation profiles in the human genome. Nat Struct Mol Biol. 2009:16(5):564-71.

17. Hu M, et al. Regulatory T cells in kidney disease and transplantation. Kidney Int. 2016;90(3):502-14.

18. Ji H, et al. Comprehensive methylome map of lineage commitment from haematopoietic progenitors. Nature. 2010;467(7313):338-42.

19. Baron $U$, et al. DNA demethylation in the human FOXP3 locus discriminates regulatory $T$ cells from activated FOXP3(+) conventional T cells. Eur J Immunol. 2007;37(9):2378-89.

20. Braza F, et al. Regulatory T cells in kidney transplantation: new directions? Am J Transplant. 2015;15(9):2288-300.

21. Hilbrands $R$, et al. Regulatory T cells and transplantation tolerance. Immunother. 2013;5(7):717-31.

22. Parker MD, et al. Ischemia- reperfusion injury and its influence on the epigenetic modification of the donor kidney genome. Transplantation. 2008;86(12):1818-23.

23. Pratt JR, et al. Ischemic epigenetics and the transplanted kidney. Transplant Proc. 2006;38(10):3344-6.

24. Zhao Y, et al. Genome-wide DNA methylation analysis in renal ischemia reperfusion injury. Gene. 2017;610:32-43.

25. Stenvinkel $P$, et al. Impact of inflammation on epigenetic DNA methylation: a novel risk factor for cardiovascular disease? J Intern Med (GBR). 2007;261(5):488-99.

26. Bechtel $W$, et al. Methylation determines fibroblast activation and fibrogenesis in the kidney. Nat Med. 2010;16(5):544-50.

27. Tampe B, et al. Tet3-mediated hydroxymethylation of epigenetically silenced genes contributes to bone morphogenic protein 7-induced reversal of kidney fibrosis. J Am Soc Nephrol. 2014;25(5):905-12.

28. Ko YA, et al. Cytosine methylation changes in enhancer regions of core pro-fibrotic genes characterize kidney fibrosis development. Genome Biol. 2013;14(10):1-14

29. Xiao X, et al. Epigenetic repression of Krüppel-like factor 4 through Dnmt1 contributes to EMT in renal fibrosis. Int J Mol Med. 2015;35(6):1596-602.

30. Larkin BP, et al. DNA methylation and the potential role of demethylating agents in prevention of progressive chronic kidney disease. FASEB J. 2018;32(10):5215-26.

31. Horvath S. DNA methylation age of human tissues and cell types. Genome Biol. 2013;14(10):R115.

32. Hannum $\mathrm{G}$, et al. Genome-wide methylation profiles reveal quantitative views of human aging rates. Mol Cell. 2013:49(2):359-67.

33. Zbieć-Piekarska R, et al. Development of a forensically useful age prediction method based on DNA methylation analysis. Forensic Sci Int Genet. 2015;17:173-9.

34. Bontha SV, et al. Systems biology in kidney transplantation: the application of multi-omics to a complex model. Am J Transplant. 2017;17(1):11-21.

35. Moran S, Arribas C, Esteller M. Validation of a DNA methylation microarray for $850,000 \mathrm{CpG}$ sites of the human genome enriched in enhancer sequences. Epigenomics. 2016:8(3):389-99.

36. Moher D, et al. Preferred reporting items for systematic reviews and meta-analyses: the PRISMA statement. PLoS Med. 2009:6(7):e1000097.

37. Liberati A, et al. The PRISMA statement for reporting systematic reviews and meta-analyses of studies that evaluate health care interventions: explanation and elaboration. J Clin Epidemiol. 2009;62(10):e1-34.

38. Wells GA, et al. The Newcastle-Ottawa Scale (NOS) for assessing the quality of nonrandomised studies in meta-analyses. Ottawa: Ottawa Hospital Research Institute; 2000.

39. Modesti PA, et al. Panethnic differences in blood pressure in Europe: a systematic review and meta-analysis. PLOS ONE. 2016;11(1):e0147601. 
40. Jadad AR, et al. Assessing the quality of reports of randomized clinical trials: Is blinding necessary? Controlled Clin Trials. 1996;17(1):1-12.

41. Mehta TK, et al. Quantitative detection of promoter hypermethylation as a biomarker of acute kidney injury during transplantation. Transplant Proc. 2006;38(10):3420-6.

42. Bontha SV, et al. Effects of DNA methylation on progression to interstitial fibrosis and tubular atrophy in renal allograft biopsies: a multi-omics approach. Am J Transplant. 2017;17(12):3060-75.

43. McGuinness $D$, et al. A molecular signature for delayed graft function. Aging Cell. 2018;17(5):e12825.

44. Heylen L, et al. Ischemia-induced DNA hypermethylation during kidney transplant predicts chronic allograft injury. J Am Soc Nephrol. 2018:29(5):1566-76.

45. Heylen $\mathrm{L}$, et al. Age-related changes in DNA methylation affect renal histology and post-transplant fibrosis. Kidney Int. 2019;96(5):1195-204

46. Schaenman J, et al. DNA Methylation age is more closely associated with infection risk than chronological age in kidney transplant recipients. Transplant Direct. 2020;6(8):e576.

47. Bestard $\mathrm{O}$, et al. Intragraft regulatory $\mathrm{T}$ cells in protocol biopsies retain Foxp3 demethylation and are protective biomarkers for kidney graft outcome. Am J Transplant. 2011;11(10):2162-72.

48. Bouvy AP, et al. The impact of induction therapy on the homeostasis and function of regulatory T cells in kidney transplant patients. Nephrol Dial Transplant. 2014;29(8):1587-97.

49. Braza F, et al. Central Role of CD45RA- Foxp $3^{\text {hi }}$ memory regulatory $T$ cells in clinical kidney transplantation tolerance. J Am Soc Nephrol. 2015:26(8):1795-805.

50. Sherston SN, et al. Demethylation of the TSDR is a marker of squamous cell carcinoma in transplant recipients. Am J Transplant. 2014;14(11):2617-22.

51. Boer $\mathrm{K}$, et al. Variations in DNA methylation of interferon gamma and programmed death 1 in allograft rejection after kidney transplantation. Clin Epigenet. 2016;8:116-116.

52. Trojan K, et al. IFNy + and IFNy - Treg subsets with stable and unstable Foxp3 expression in kidney transplant recipients with good long-term graft function. Transplant Immunol. 2016;39:1-9.

53. Trojan K, et al. Helios expression and Foxp3 TSDR methylation of IFNy+ and IFNy-Treg from kidney transplant recipients with good longterm graft function. PLOS ONE. 2017;12(3):e0173773.

54. Alvarez Salazar EK, et al. Methylation of FOXP3 TSDR underlies the impaired suppressive function of tregs from long-term belatacepttreated kidney transplant patients. Front Immunol. 2017:8:219.

55. Peters FS, et al. Differentially methylated regions in T cells identify kidney transplant patients at risk for de novo skin cancer. Clin Epigenet. 2018;10:81.

56. Peters FS, et al. Disrupted regulation of serpinB9 in circulating T cells is associated with an increased risk for post-transplant skin cancer. Clin Exp Immunol. 2019;197(3):341-51.

57. Cortés-Hernández A, et al. Ex vivo expansion of regulatory T cells from long-term Belatacept-treated kidney transplant patients restores their phenotype and suppressive function but not their FOXP3 TSDR demethylation status. Cell Immunol. 2020;348:104044.

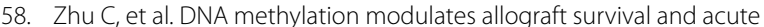
rejection after renal transplantation by regulating the mTOR pathway. Am J Transplant. 2021;21(2):567-81.

59. Soyoz $M$, et al. Consideration of IL-2, IFN- $\gamma$ and IL-4 expression and methylation levels in CD4+ T cells as a predictor of rejection in kidney transplant. Transplant Immunol. 2021;68:101414.

60. Rodriguez RM, et al. Defining a methylation signature associated with operational tolerance in kidney transplant recipients. Front Immunol. 2021;12:3352.

61. Wieczorek G, et al. Quantitative DNA methylation analysis of FOXP3 as a new method for counting regulatory $T$ cells in peripheral blood and solid tissue. Cancer Res. 2009:69(2):599-608.

62. Martin-Moreno PL, Tripathi S, Chandraker A. Regulatory T cells and kidney transplantation. Clin J Am Soc Nephrol. 2018;13(11):1760-4.

63. Schübeler D. Function and information content of DNA methylation. Nature. 2015;517(7534):321-6.

64. McCaughan JA, et al. Epigenetics: time to translate into transplantation. Transplantation. 2012;94(1):1-7.
65. Obata Y, Furusawa Y, Hase K. Epigenetic modifications of the immune system in health and disease. Immunol Cell Biol. 2015;93(3):226-32.

66. Menke J, et al. The effect of ischemia/reperfusion on the kidney graft. Curr Opin Organ Transplant. 2014;19(4):395-400.

67. Cravedi P, Heeger PS. Complement as a multifaceted modulator of kidney transplant injury. J Clin Investig. 2014;124(6):2348-54.

68. Ponticelli C. Ischaemia-reperfusion injury: a major protagonist in kidney transplantation. Nephrol Dial Transplant. 2014;29(6):1134-40.

69. Wing $M R$, et al. DNA methylation profile associated with rapid decline in kidney function: findings from the CRIC study. Nephrol Dial Transplant. 2014;29(4):864-72.

70. Hosgood SA, et al. The use of ex-vivo normothermic perfusion for the resuscitation and assessment of human kidneys discarded because of inadequate in situ perfusion. J Transl Med. 2015;13:329.

71. Atianand MK, Fitzgerald KA. Long non-coding RNAs and control of gene expression in the immune system. Trends Mol Med. 2014;20(11):623-31.

72. Weng N-P, Araki Y, Subedi K. The molecular basis of the memory T cell response: differential gene expression and its epigenetic regulation. Nat Rev Immunol. 2012;12(4):306-15.

73. Villard J. The role of natural killer cells in human solid organ and tissue transplantation. J Innate Immun. 2011;3(4):395-402.

74. Ogbomo H, et al. Histone deacetylase inhibitors suppress natural killer cell cytolytic activity. FEBS Lett. 2007;581(7):1317-22.

75. Li G, et al. Epigenetic regulation of killer immunoglobulin-like receptor expression in T cells. Blood. 2009;114(16):3422-30.

76. Sakaguchi $\mathrm{S}$, et al. Immunologic self-tolerance maintained by activated T cells expressing IL-2 receptor alpha-chains (CD25). Breakdown of a single mechanism of self-tolerance causes various autoimmune diseases. J Immunol. 1995;155(3):1151-64.

77. Hall BM, et al. Specific unresponsiveness in rats with prolonged cardiac allograft survival after treatment with cyclosporine. III. Further characterization of the CD4+ suppressor cell and its mechanisms of action. J Exp Med. 1990;171(1):141-57.

78. Fontenot JD, Gavin MA, Rudensky AY. Foxp3 programs the development and function of CD4+CD25+ regulatory T cells. Nat Immunol. 2003:4(4):330-6.

79. Khattri $\mathrm{R}$, et al. An essential role for Scurfin in CD4+CD25+ T regulatory cells. Nat Immunol. 2003;4(4):337-42.

80. Hori S, Nomura T, Sakaguchi S. Control of regulatory T cell development by the transcription factor Foxp3. Science. 2003;299(5609):1057-61.

81. Heylen $\mathrm{L}$, et al. The emerging role of DNA methylation in kidney transplantation: a perspective. Am J Transplant. 2016;16(4):1070-8.

82. Baron $U$, et al. DNA demethylation in the human FOXP3 locus discriminates regulatory $T$ cells from activated FOXP3+ conventional T cells. Eur J Immunol. 2007;37(9):2378-89.

83. Lal $\mathrm{G}$, et al. Epigenetic regulation of Foxp3 expression in regulatory $T$ cells by DNA methylation. J Immunol. 2009;182(1):259-73.

84. Toker A, et al. Active demethylation of the Foxp3 locus leads to the generation of stable regulatory T cells within the thymus. J Immunol. 2013;190(7):3180-8.

85. Shevach EM. Mechanisms of foxp3+T regulatory cell-mediated suppression. Immunity. 2009;30(5):636-45.

86. Wang $Z$, et al. Prospects of the use of cell therapy to induce immune tolerance. Front Immunol. 2020;11:792.

87. Jeffries MA. Epigenetic editing: how cutting-edge targeted epigenetic modification might provide novel avenues for autoimmune disease therapy. Clin Immunol. 2018;196:49-58.

88. Chen S, et al. Epigenetically modifying the Foxp3 locus for generation of stable antigen-specific Tregs as cellular therapeutics. Am J Transplant. 2020;20(9):2366-79

89. Lasseigne BN, Brooks JD. The role of DNA methylation in renal cell carcinoma. Mol Diagn Ther. 2018;22(4):431-42.

90. Is the dose right? Adverse Drug React Bull. 1989:135:504-507.

91. Boer K, Hesselink DA, Baan CC. Variations in DNA methylation and allograft rejection. Curr Opin Organ Transplant. 2021;26(1):30-6.

92. Dor Y, Cedar H. Principles of DNA methylation and their implications for biology and medicine. Lancet. 2018;392(10149):777-86.

93. Lehmann-Werman $\mathrm{R}$, et al. Monitoring liver damage using hepatocytespecific methylation markers in cell-free circulating DNA. JCI Insight. 2018:3(12):e120687. 
94. Oellerich $\mathrm{M}$, et al. Absolute quantification of donor-derived cell-free DNA as a marker of rejection and graft injury in kidney transplantation: results from a prospective observational study. Am J Transplant. 2019;19(11):3087-99.

95. Gielis EM, et al. The use of plasma donor-derived, cell-free DNA to monitor acute rejection after kidney transplantation. Nephrol Dial Transplant. 2020;35(4):714-21.

96. Watson $\mathrm{D}$, et al. A novel multi-biomarker assay for non-invasive quantitative monitoring of kidney injury. J Clin Med. 2019;8(4):499.

97. Yang JYC, et al. A urine score for noninvasive accurate diagnosis and prediction of kidney transplant rejection. Sci Transl Med. 2020;12(535):eaba2501.

98. Maggiore U, Leventhal J, Cravedi P. Rethinking clinical endpoints in kidney transplant trials. Curr Opin Organ Transplant. 2020;25(1):1-7.

99. Yong W-S, Hsu F-M, Chen P-Y. Profiling genome-wide DNA methylation. Epigenet Chromatin. 2016;9(1):26.

100. Irizarry RA, et al. Comprehensive high-throughput arrays for relative methylation (CHARM). Genome Res. 2008;18(5):780-90.

101. Taiwo O, et al. Methylome analysis using MeDIP-seq with low DNA concentrations. Nat Protoc. 2012;7(4):617-36.

102. Mallik S, et al. An evaluation of supervised methods for identifying differentially methylated regions in Illumina methylation arrays. Brief Bioinform. 2018;20(6):2224-35.

103. Bibikova M, et al. High density DNA methylation array with single CpG site resolution. Genomics. 2011;98(4):288-95.

104. Urich MA, et al. MethylC-seq library preparation for base-resolution whole-genome bisulfite sequencing. Nat Protoc. 2015;10(3):475-83.

105. Meissner A, et al. Genome-scale DNA methylation maps of pluripotent and differentiated cells. Nature. 2008;454(7205):766-70.

106. Pidsley $R$, et al. Critical evaluation of the Illumina MethylationEPIC BeadChip microarray for whole-genome DNA methylation profiling. Genome Biol. 2016;17(1):208.

107. Solomon O, et al. Comparison of DNA methylation measured by Illumina 450K and EPIC BeadChips in blood of newborns and 14-year-old children. Epigenetics. 2018;13(6):655-64.

108. Wang Z, Wu X, Wang Y. A framework for analyzing DNA methylation data from Illumina Infinium HumanMethylation450 BeadChip. BMC Bioinform. 2018;19(5):115.

109. How Kit A, Nielsen HM, Tost J. DNA methylation based biomarkers: practical considerations and applications. Biochimie. 2012;94(11):2314-37.

110. Jaffe $A E$, et al. Bump hunting to identify differentially methylated regions in epigenetic epidemiology studies. Int J Epidemiol. 2012:41(1):200-9.

111. Silva T, et al. TCGA Workflow: analyze cancer genomics and epigenomics data using Bioconductor packages [version 2; peer review: 1 approved, 2 approved with reservations]. F1000Research. 2016;5:1542.

112. Aryee MJ, et al. Minfi: a flexible and comprehensive Bioconductor package for the analysis of Infinium DNA methylation microarrays. Bioinformatics. 2014;30(10):1363-9.

113. Rauschert S, et al. Machine learning and clinical epigenetics: a review of challenges for diagnosis and classification. Clin Epigenet. 2020;12(1):51.

114. LeCun Y, Bengio Y, Hinton G. Deep learning. Nature. 2015:521(7553):436-44.

115. Galanter JM, et al. Differential methylation between ethnic sub-groups reflects the effect of genetic ancestry and environmental exposures. eLife. 2017;6:e20532.

116. Greene T, et al. Performance of GFR slope as a surrogate end point for kidney disease progression in clinical trials: a statistical simulation. J Am Soc Nephrol. 2019;30(9):1756-69.

117. R Core Team. R: a language and environment for statistical computing R Foundation for Statistical Computing. 2021.

118. Davis S, et al. Methylumi: handle illumina methylation data. R Package version 2.0. 2014.

119. Teschendorff $A E$, et al. A beta-mixture quantile normalization method for correcting probe design bias in illumina infinium 450 k DNA methylation data. Bioinformatics. 2012;29(2):189-96.

120. Niu L, Xu Z, Taylor JA. RCP: a novel probe design bias correction method for illumina methylation beadchip. Bioinformatics. 2016;32(17):2659-63.

121. Phipson B, Maksimovic J, Oshlack A. missMethyl: an R package for analyzing data from Illumina's HumanMethylation450 platform. Bioinformatics. 2015;32(2):286-8.
122. Levy JJ, et al. MethylNet: an automated and modular deep learning approach for DNA methylation analysis. BMC Bioinform. 2020;21(1):108.

\section{Publisher's Note}

Springer Nature remains neutral with regard to jurisdictional claims in published maps and institutional affiliations.
Ready to submit your research? Choose BMC and benefit from:

- fast, convenient online submission

- thorough peer review by experienced researchers in your field

- rapid publication on acceptance

- support for research data, including large and complex data types

- gold Open Access which fosters wider collaboration and increased citations

- maximum visibility for your research: over 100M website views per year

At BMC, research is always in progress.

Learn more biomedcentral.com/submissions 